\title{
Study and modelling of droop-controlled islanded mesh microgrids
}

\author{
Youssef Hennane ${ }^{1,2, *}$, Abdelmajid Berdai $^{2}$, Serge Pierfederici ${ }^{2}$, Farid Meibody-Tabar ${ }^{1}$, and Vitaliy Kuznetsov ${ }^{3}$ \\ ${ }^{1}$ Université de Lorraine, CNRS, LEMTA, F-54000 Nancy, France \\ ${ }^{2}$ CEDoc Sciences de l'Ingénieur ENSEM, LESE, Route d'El Jadida, km 7, BP: 8118, Oasis, Casablanca, Maroc \\ ${ }^{3}$ National Metallurgical Academy of Ukraine, Department of the electrical engineering and electromechanics, Dnipro, Ukraine
}

\begin{abstract}
The active and reactive power sharing of distributed generation sources (DGs) connected to isolated microgrids with a single point of common coupling (mono-PCC) to which the loads are also connected has already been the subject of several studies. A high penetration rate of DGs based on renewable energies has as a logical consequence the development and implementation of mesh and more complex multiPCC microgrids. In this paper, a developed droop control method for synchronization and power sharing between different DGs connected to a mesh islanded multi-PCC microgrid with many distributed generation sources (DGs) and different type of loads (including active load (CPL)) randomly connected to different PCCs is applied. Then, a state model of the entire mesh microgrid is developed integrating the generators with their controllers, power lines, droop algorithms and dynamic loads. This model is then used to study the asymptotic stability and robustness properties of the system. The simulation results confirm the effectiveness of the applied strategies for the synchronization of the different DGs to the microgrid while ensuring an efficient active and reactive power sharing. also, they confirm the validity of the developed state space model of the system.
\end{abstract}

\section{Introduction}

Microgrids powered by clean renewable energy through distributed power generation units are one of many solutions to reduce greenhouse gas emissions [1]. The intermittency of renewable energies can lead to some serious microgrid instability problems. therefore, one of the challenges is to synchronize and connect all distributed generators to an isolated microgrid while ensuring "plug and play" functionality and respecting the active and reactive power sharing between the different distributed generation units [2-4].

Strategies based on Droop control is currently the most used methods for power sharing and synchronization in literature strategy [5-9]. Those strategies were proven to be effective in microgrids with a single point of common coupling (mono-PCC) like in [10].

Unlike in mono-PCC microgrids those strategies for synchronization and power sharing are less efficient when they are applied on a mesh multi-PCC microgrids, with many distributed generation sources (DGs) and different type of loads randomly connected to different PCCs. And that's firstly because of the strong couplings between active and reactive powers injected by the DGs in different microgrid PCCs introduced by the line feeders and also due to the complex nature of the mesh microgrid.

Limited researches have focused on solutions that concern the active and reactive power sharing in mesh microgrids [10-12]. In [10] the authors propose the use of bi-directional communication. In [11] a variable Droop control strategy with virtual impedance correction loop and [12] using a modified Droop control strategy by adding a decoupling term in order to remove the coupling phenomenon between active and reactive power.

Another problem is the possible unstable behavior of the microgrid due to the interaction between the DGs and loads, especially the ones supplied trough tightly regulated power converters. These loads behave as constant power loads (CPL) and may cause the system instability [13-15].

In this paper, a mesh microgrid constituted by several PCCs to which are connected multiple DGs and different type of loads including an active load CPL is considered. The different PCCs are interconnected with power supply lines, modeled by RLC circuits inspired by an IEEE 9-bus test feeder Fig. 2. Like as in [12], a modified droop control and synchronization strategies, adapted to the considered multi-PCC mesh microgrid is applied in order to ensure an accurate power sharing and to provide the "plug and play" function. The microgrid voltage and frequency are imposed by the first DG connected. For other DGs an adapted synchronization strategy is proposed and applied before its interconnection to the microgrid.

To study the stability of the mesh microgrid and the robustness of its control, a state model of the entire mesh microgrid is developed considering the generators with their decentralized controllers integrating modified droop

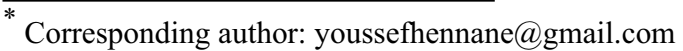


algorithms, power lines and several loads including a CPL.

The simulation results confirm the effectiveness of the applied strategies and the validity of the developed mathematical model.

\section{Power sharing strategies in mesh multi-PCC islanded microgrids}

Classical Droop control strategy in (1) and (2) can be efficient for power sharing in mono-PCC microgrids in Fig. 1 if and only if the microgrid lines impedances were ignored. Therefore, a developed Droop control strategy in (3) and (4) from [11] that was proven efficient for power sharing in mono-PCC microgrids is applied on the mesh multi-PCC islanded microgrid in Fig.2.

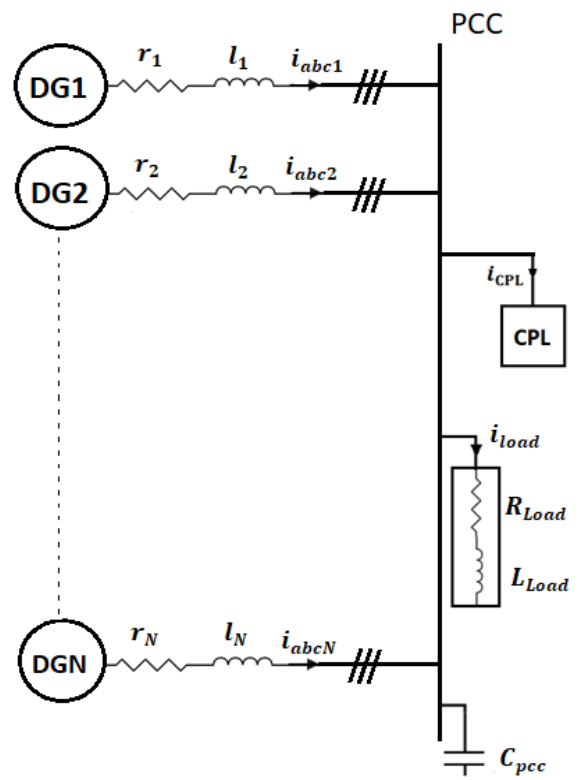

Fig. 1. Mono-PCC microgrid.

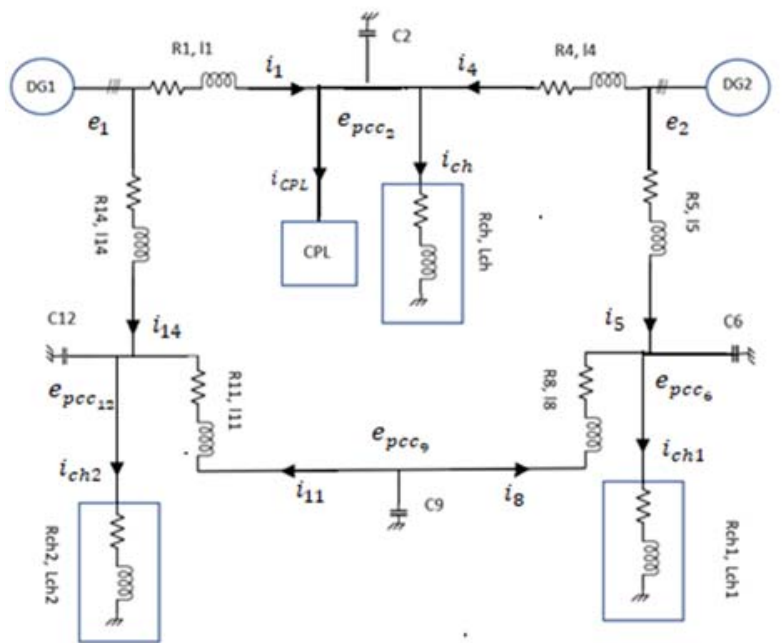

Fig. 2. Mesh multi-PCC microgrid.

$$
\begin{aligned}
& \omega_{i}=\omega_{n}-m_{i}\left(P_{i}-P_{i n}\right) \\
& V_{i}=V_{n}-n_{i}\left(Q_{i}-Q_{i n}\right)
\end{aligned}
$$

$$
\text { With : } \quad m_{i}=\frac{\Delta_{\omega}}{P_{\text {in }}}, \quad n_{i}=\frac{\Delta_{V}}{Q_{\text {in }}}
$$

Classical Droop control in (1) and (2) sets for each DG, the frequency and the voltage amplitude at the associated PCC. Where $P_{i}$ and $Q_{i}$ are the measured values of the active and reactive power of the $i^{\text {th }} \mathrm{DG}, \mathrm{P}_{\text {in }}$ and $\mathrm{Q}_{\text {in }}$ are their nominal values, $\omega n$ and $V_{n}$ are the nominal values of the pulse and voltage of the $i^{\text {th }} \mathrm{DG}, \Delta_{\omega}$ and $\Delta_{\mathrm{V}}$ are the permissible variations of the pulse and voltage, and $\mathrm{m}_{\mathrm{i}}$ and $\mathrm{n}_{\mathrm{i}}$ are the droop control coefficients.

$$
\begin{aligned}
& \delta_{i}=\int\left(K_{a}\left(\delta_{i n}-\delta_{L}\right)-m_{i}\left(P_{i}-P_{i n}\right)\right) \cdot d t \\
& V_{i}=\int\left(K_{e}\left(V_{\text {in }}-V_{L}\right)-n_{i}\left(Q_{i}-Q_{i n}\right)\right) \cdot d t
\end{aligned}
$$

The proposed angular droop aims to indirectly control the voltage at the PCC and its phase to be equal to the nominal values (i.e. $\mathrm{V}_{\text {in }}$ and $\delta_{\text {in }}$ ). The added integrators can minimize the static error between the return signal and the corresponding nominal values. By choosing identical $\mathrm{K}_{\mathrm{a}}$ and $\mathrm{K}_{\mathrm{e}}$ for each generator, a precise active and reactive power sharing is achieved that no longer depends on the system impedance

It can be observed in Fig. 3 that the proposed strategy in (3) and (4) ensure a perfect active power sharing while Fig.4 prove that the applied strategy does not guarantee an efficient reactive power sharing due to the complexity of the microgrid.

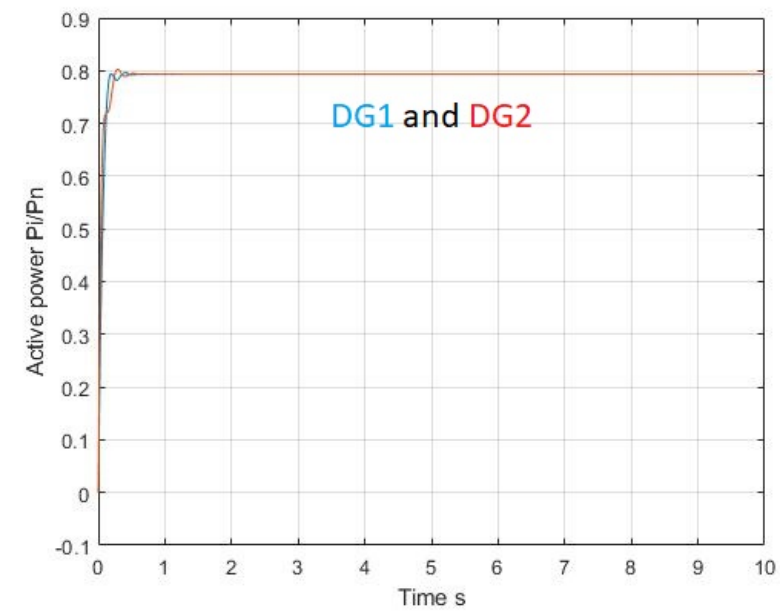

Fig. 3. Evolution of the DGs active powers (both DGs are connected to the microgrid at $0 \mathrm{~s}$ ).

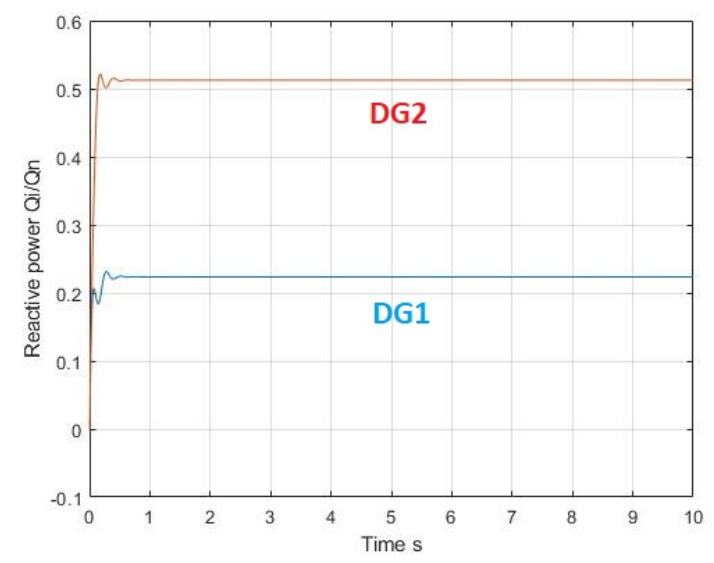

Fig. 4. Evolution of the DGs reactive powers (both DGs are connected to the microgrid at $0 \mathrm{~s}$ ). 
In multi-PCC microgrids, each line connecting the $\mathrm{i}^{\text {th }} \mathrm{PCC}$ to the $\mathrm{j}^{\text {th }}$ PCC has a non-negligible inductance $\lambda_{i, j}$ and a resistor $\rho_{i, j}$. Due to this phenomenon, the line voltage drop between these two PCCs creates a coupling between the active $\left(P_{i, j}\right)$ and reactive $\left(Q_{i, j}\right)$ powers exchanged [12], according to the following formula (5):

$\Delta V=\rho_{i, j} I_{i, j} \cos \varphi+j \lambda_{i, j} \omega I_{i, j} \sin \varphi=\frac{\rho_{i, j} P_{i, j}+j \lambda_{i, j} Q_{i, j}}{V_{i}}(5)$

With $\varphi$ the phase shift between the voltage $V_{i}$ and the current $I_{i, j}$.
Based on the strategies developed in [12] to obtain an efficient reactive power sharing in this type of multi-PCC microgrids, the voltage equation is modified by adding a decoupling term (see equation (7)) removing the coupling phenomenon between active and reactive power. This non-linear coefficient, called $\mathrm{J}_{\mathrm{i}}$, is estimated using a PI regulator, forcing the cancellation of the $\varepsilon_{\mathrm{i}}$ error defined in (8).

In steady state, when the error $\varepsilon_{\mathrm{i}}$ tends towards zero, the reactive power is shared between the DGs.

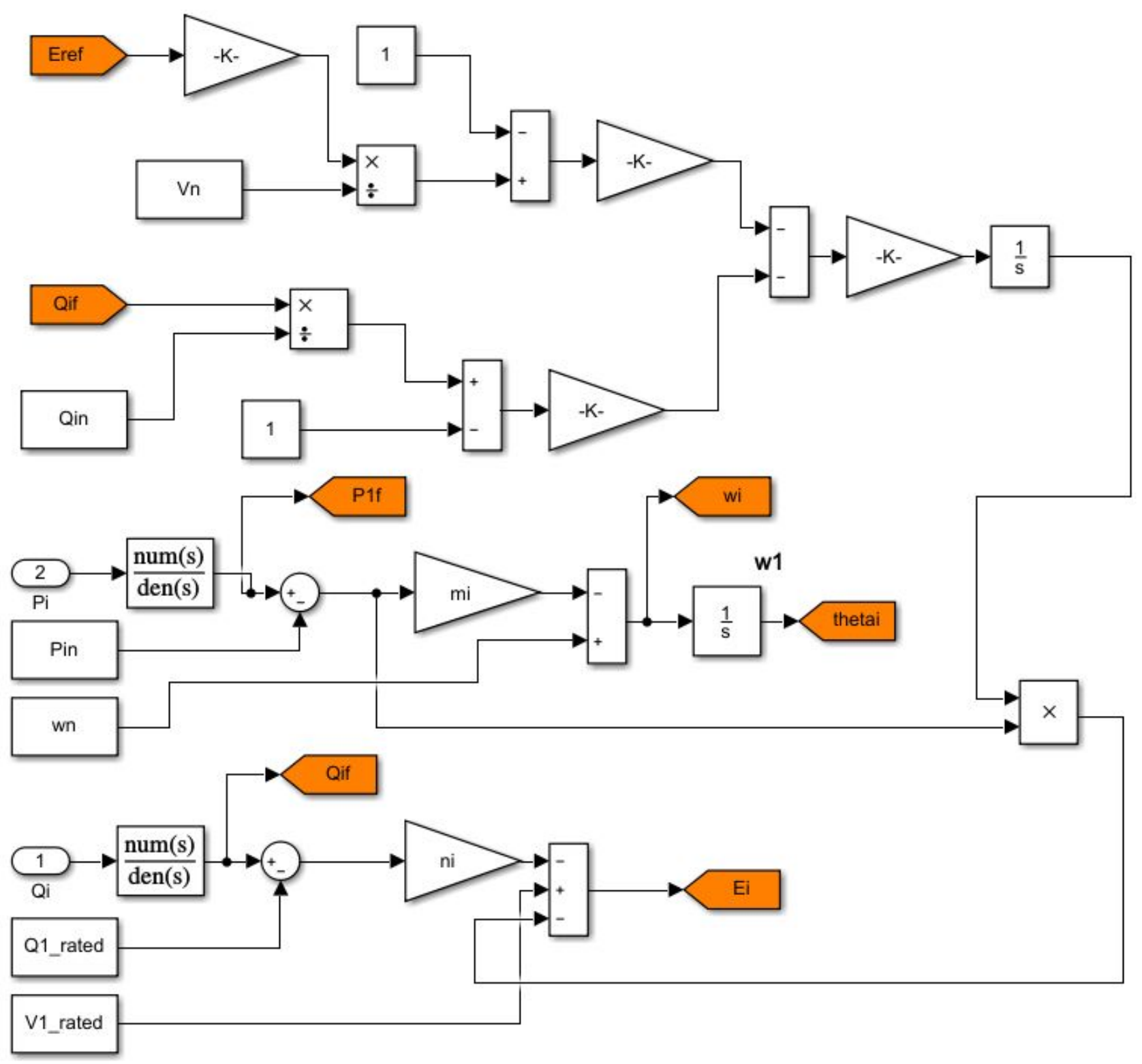

Fig. 5. Primary control.

This approach allows the primary control of the voltage of the $\mathrm{i}^{\text {th }}$ DG Fig.5, regardless of the operating point of the loads. It should be noted that $\mathrm{V}_{\text {ref }}$ in Fig. 5 and equation (7) is a reference potential corresponding to one of the PCC voltages in the studied mesh Microgrid serving as a pilot node and its nominal voltage is called $\mathrm{V}_{\mathrm{n}}$.

$$
\begin{aligned}
& \omega_{i}=\omega_{0}-m_{i}\left(P_{i}-P_{i n}\right) \\
& V_{i}=V_{0}-n_{i}\left(Q_{i}-Q_{i n}\right)-J_{i}\left(P_{i}-P_{i n}\right)
\end{aligned}
$$


With: $\quad J_{i}=K_{P} \varepsilon_{i}+K_{I} \int \varepsilon_{i} d t$

$$
\varepsilon_{i}=\left[-\left(\frac{V_{r e f}}{V_{n}}-1\right)-\left(\frac{Q_{i}}{Q_{\text {in }}}-1\right)\right]
$$

In the absence of information on $\mathrm{V}_{\text {ref }}$ the coefficient $\mathrm{J}$ is set to 0 and we return to the classical droop in (1) and (2).

\section{Synchronization strategy in mesh multi-PCC islanded microgrids}

Due to the complexity of the mesh multi-PCC microgrid and the intermittency of renewable energy, DGs frequently connect and disconnect from the microgrid which make the integration of a fast and an efficient synchronization strategy an obligation. The big challenge here is to propose a synchronization strategy that is at the same time efficient and doesn't affect the power sharing between the DGs already connected to the microgrid.

To show the effect of the lack of synchronization in mesh multi-PCC islanded microgrid, modified droop control in (3) and (4) is applied to the mesh microgrid.

It can be seen in Fig. 6 that the strategy in (3) and (4) as explained in section 2 does ensure a perfect active power sharing but at $5 \mathrm{~s}$ it can be remarked that an unacceptable disturbing active power peak occur due to the lack of synchronization.

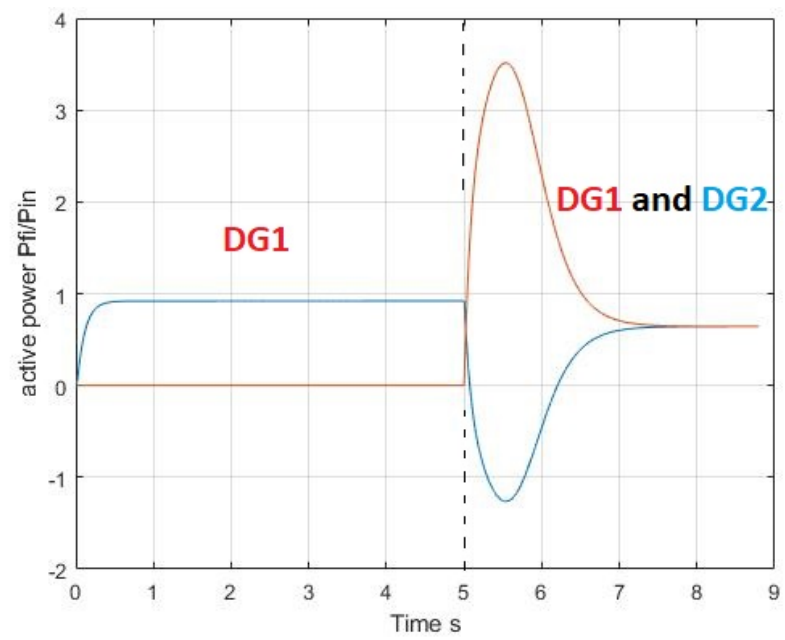

Fig. 6. Evolution of the DG active powers (first DG supplies the microgrid up to $5 \mathrm{~s}$, the second DG is connected to the microgrid at $5 \mathrm{~s}$ ).
The same power peak occurs in the DG's reactive powers.

To remove those unacceptable power peaks, a fast and an efficient synchronization of the $i^{\text {th }}$ DG to the $i^{\text {th }}$ PCC should be applied before their interconnection. Therefore, the voltage amplitude $\mathrm{V}_{p c c i}$, pulsation $\omega_{p c c i}$ and phase $\theta_{p c c i}$ of the $i^{\text {th }}$ PCC must be approximately equal to those of the $\mathrm{i}^{\text {th }} \mathrm{DG}\left(\mathrm{V}_{D G i}, \omega_{D G i}, \theta_{D G i}\right)$. To achieve this objective, the errors between the amplitudes, pulsations and phases of both sides (the $i^{\text {th }}$ DG and the $i^{\text {th }}$ PCC) are forced to zero by adding only during the synchronization interval pure integral controllers to the $i^{\text {th }}$ DG Droop control as shown in equations (9) and (10) [12]

$$
\begin{aligned}
\omega_{i}= & \omega_{0}-\mathrm{m}_{i}\left(\mathrm{P}_{i}-\mathrm{P}_{i n}\right)+K_{a i} \int\left(\omega_{p c c i}-\right. \\
& \left.\omega_{D G i}\right)-K_{b i} \int\left(\theta_{D G i}-\theta_{p c c i}\right) \\
\mathrm{V}_{i}= & \mathrm{V}_{0}-\mathrm{n}_{i}\left(\mathrm{Q}_{i}-\mathrm{Q}_{i n}\right)-\mathrm{J}_{i}\left(\mathrm{P}_{i}-\mathrm{P}_{i n}\right)-K_{e i} \int\left(V_{D G i}-\right. \\
& \left.V_{p c c i}\right)
\end{aligned}
$$

\section{System modeling}

Due to the dynamic interaction between the DGs and the loads, the stability of the microgrids is strongly influenced. Therefore, for a future study of system stability and robustness, a complete mathematical modeling of the system is provided in this section.

\subsection{State-space model of a distributed generator (DG)}

In order to formulate the equations describing a $\mathrm{DGi}$, an equivalent synoptic diagram is shown in Fig. 7.

- Power calculation

As shown in Figure 7, the measured output voltages and currents are used to calculate the instantaneous active and reactive powers using the equations (11):

$$
\left\{\begin{array}{c}
P_{i}=e_{d i} * i_{d i}+e_{q i} i_{q i} \\
Q_{i}=e_{q i} * i_{d i}-e_{d i} * i_{q i}
\end{array}\right.
$$

- Low-pass filter

To obtain the average values $P_{f i}$ and $Q f_{i}$ The instantaneous powers $P_{i}$ and $Q_{i}$ pass through a first order low-pass filter in (12), with $\omega_{f}$ representing its filter cutoff frequency.

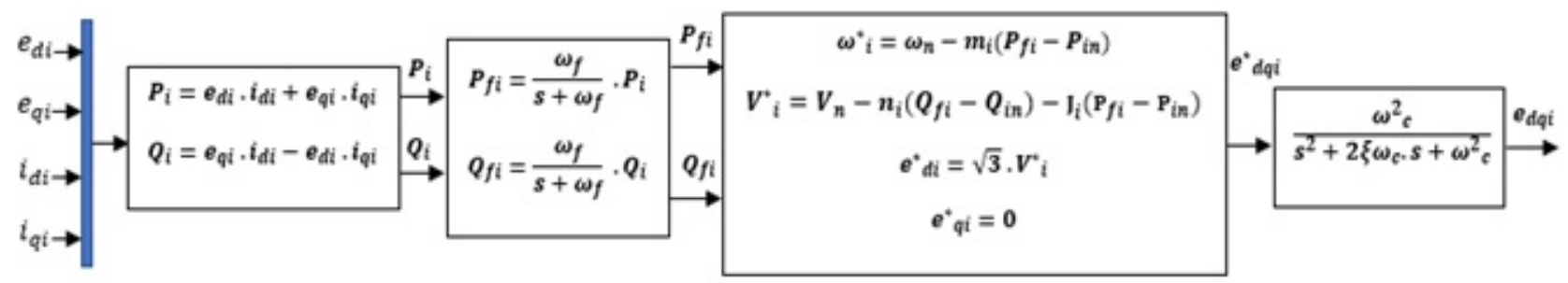

Power caculation Low-pass filter

Fig. 7. Equivalent synoptic diagram describing a Droop controlled $\mathrm{DG}_{\mathrm{i}}$. 


$$
\left\{\begin{array}{l}
\frac{d}{d t} P_{f i}=\omega_{f}\left(P_{i}-P_{f i}\right) \\
\frac{d}{d t} Q_{f i}=\omega_{f}\left(Q_{i}-Q_{f i}\right)
\end{array}\right.
$$

\section{- Droop control}

The third bloc applies the modified Droop control strategy in (6) and (7)

- Inner control

The fourth bloc models the delay imposed by the Voltage Source Inverter (VSI) controlling the $i^{\text {th }}$ DG output voltages by means of a second-order filter. This filter which has a faster dynamic compared to the external droop control loop allows the output voltages $i^{\text {th }}$ DG $\left(e^{*} i_{d q}\right)$ following their voltage references $\left(e_{i_{d q}}\right)$. The transfer function of this forth bloc is defined in (13):

$$
\frac{e_{i d q}}{e^{*} i_{d q}}=\frac{\omega^{2} c}{s^{2}+2 \xi \omega_{c} \cdot s+\omega^{2} c}
$$

\subsection{Microgrid structure and modelling}

In this section the microgrid used for modelling is the one presented in section 2. The considered Microgrid is composed of two DGs powering three classical inductive loads, modeled by serial R-L circuits, and a CPL load. They are interconnected by RLC power lines to present a mesh multi-PCC microgrid.

In order to study the considered microgrid having 2 DGs, the state equations are given using a common reference frame for expressing the different variables. The used d-q reference frame is defined in a way that its d-axis is oriented toward the first $\mathrm{DG}$ voltage vector $\mathrm{V}_{1}$ (Fig.8). In steady state, the frequencies of the system are the same (i.e. $\omega_{i}=\omega_{j}=\omega_{\text {com }}$ ).

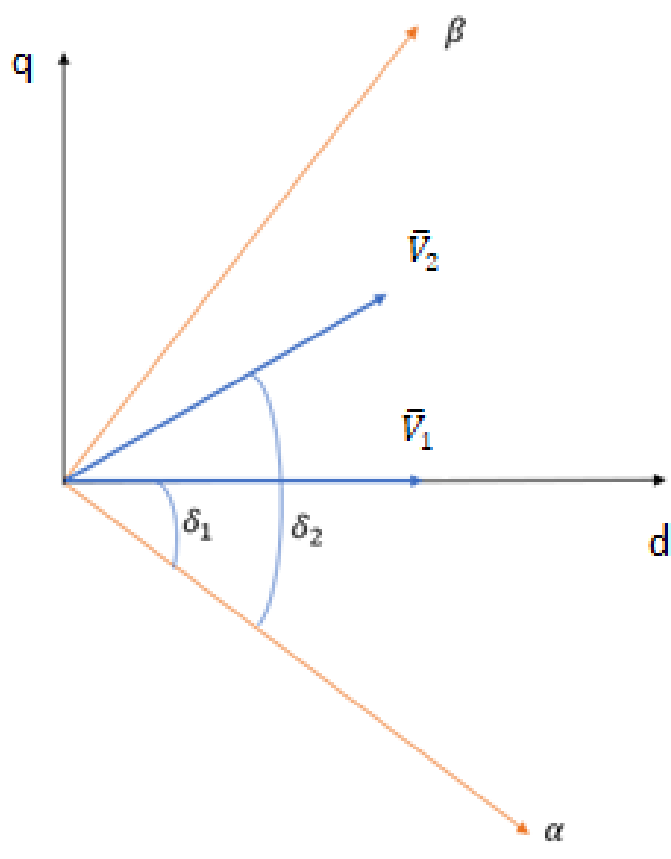

Knowing that the phase voltages of DG1 and DG2 (connected to PCC1 and PCC2) have the RMS values of $\boldsymbol{V}_{\mathbf{1}}$ and $\boldsymbol{V}_{2}$, the $\boldsymbol{\alpha}-\boldsymbol{\beta}$ components of $\overline{\boldsymbol{V}}_{\mathbf{1}}$ and $\overline{\boldsymbol{V}}_{\mathbf{2}}$ are obtained using Concordia transformation $\boldsymbol{T}_{\mathbf{3 2}}^{\boldsymbol{t}}$ :

With: $T_{32}^{t}=\sqrt{\frac{2}{3}} \cdot\left[\begin{array}{ccc}1 & -\frac{1}{2} & -\frac{1}{2} \\ 0 & \frac{\sqrt{3}}{2} & -\frac{\sqrt{3}}{2}\end{array}\right]$

$\mathrm{DG} 1:\left\{\begin{array}{l}\left(\begin{array}{l}e_{d 1} \\ e_{q 1}\end{array}\right)=P\left(-\delta_{1}\right) \cdot\left(\begin{array}{l}V_{\alpha 1} \\ V_{\beta 1}\end{array}\right) \\ \left(\begin{array}{l}e_{d 1} \\ e_{q 1}\end{array}\right)=\left(\begin{array}{cc}\cos \delta_{1} & \sin \delta_{1} \\ -\sin \delta_{1} & \cos \delta_{1}\end{array}\right) \cdot\left(\begin{array}{l}\sqrt{3} \cdot V_{1} \cdot \cos \delta_{1} \\ \sqrt{3} \cdot V_{1} \cdot \sin \delta_{1}\end{array}\right) \\ \left(\begin{array}{l}e_{d 1} \\ e_{q 1}\end{array}\right)=\left(\begin{array}{c}\sqrt{3} \cdot V_{1} \\ 0\end{array}\right)\end{array}\right.$

$\mathrm{DG} 2:\left\{\begin{array}{l}\left(\begin{array}{l}e_{d 2} \\ e_{q 2}\end{array}\right)=P\left(-\delta_{1}\right) \cdot\left(\begin{array}{l}V_{\alpha 2} \\ V_{\beta 2}\end{array}\right) \\ \left(\begin{array}{l}e_{d 2} \\ e_{q 2}\end{array}\right)=\left(\begin{array}{cc}\cos \delta_{1} & \sin \delta_{1} \\ -\sin \delta_{1} & \cos \delta_{1}\end{array}\right) \cdot\left(\begin{array}{l}\sqrt{3} \cdot V_{2} \cdot \cos \delta_{2} \\ \sqrt{3} \cdot V_{2} \cdot \sin \delta_{2}\end{array}\right) \\ \left(\begin{array}{l}e_{d 2} \\ e_{q 2}\end{array}\right)=\left(\begin{array}{l}\sqrt{3} \cdot V_{2} \cdot \cos \left(\delta_{2}-\delta_{1}\right) \\ \sqrt{3} \cdot V_{2} \cdot \sin \left(\delta_{2}-\delta_{1}\right)\end{array}\right)\end{array}\right.$

- Network model

Equations (16) present a network model of $\mathrm{N}$ supply lines, connecting $\mathrm{N}$ DGs to $\mathrm{N}$ randomly placed common coupling points (PCC) of a microgrid.

$$
\left\{\begin{array}{l}
\frac{d}{d t} i_{d_{i}}=\frac{1}{L_{i}} \Delta e_{d_{j k}}-\frac{r_{i}}{L_{i}} i_{d_{i}}+\omega_{c o m} i_{q_{i}} \\
\frac{d}{d t} i_{q_{i}}=\frac{1}{L_{i}} \Delta e_{q_{j k}}-\frac{r_{i}}{L_{i}} i_{q_{i}}-\omega_{c o m} i_{d_{i}}
\end{array}\right.
$$

With:

$$
\Delta e_{d, q_{j k}}=\left(e^{*}{ }_{d, q_{j}}-e^{*}{ }_{d, q_{k}}\right), e^{*}{ }_{d, q_{j}} \in\left\{e_{d, q_{j}}, e_{p c c}{ }_{d, q_{j}}\right\}
$$

- $\quad$ Load model

The loads considered in this work are serial passive R-L loads and an active CPL (Fig. 2). The next equations (17) are written for all PCCs except the ones connected to the DGs for which the voltages are imposed by their droop controllers.

$$
\left\{\begin{array}{l}
\frac{d}{d t} e_{p c c d_{i}}=\frac{1}{C_{p c c i}}\left(\sum i_{D i}-i_{C H d i}-i_{C P L q i}\right)++\omega_{c o m} \cdot e_{p c c q_{i}} \\
\frac{d}{d t} e_{p c c q_{i}}=\frac{1}{C_{p c c i}}\left(\sum i_{Q i}-i_{C H q i}-i_{C P L d i}\right)-\omega_{c o m} \cdot e_{p c c d_{i}}
\end{array}\right.
$$

$$
\left\{\begin{array}{l}
\frac{d}{d t} i_{C H d i}=\frac{1}{L_{C H i}}\left(e_{p c c d_{i}}-R c h_{i} \cdot i_{C H d i}\right)+\omega_{c o m} \cdot i_{C H q i} \\
\frac{d}{d t} i_{C H q i}=\frac{1}{L_{C H i}}\left(e_{p c c q_{i}}-R c h_{i} \cdot i_{C H q i}\right)-\omega_{c o m} \cdot i_{C H d i}
\end{array}\right.
$$

Fig. 8. Reference Frames $\alpha-\beta$ and $d-q$. 
With $R c h_{i}$ and $L c h_{i}$ representing the resistance and inductance of the RL load. $i_{C P L d i}$ and $i_{C P L q i}$ are the input current of the CPL load.

The active $C P L_{i}$ connected to $P C C_{i}$ is supposed to absorb the active and reactive powers $P_{C P L i}$ and $Q_{C P L i}$. They are related to the d-q components of the current absorbed by $C P L_{i}\left(i_{C P L i d}\right.$ and $\left.i_{C P L i q}\right)$ by the next relation:

$$
\left\{\begin{array}{l}
e_{C P L_{D Q i}}=\left(\begin{array}{cc}
e_{p c c d_{i}} & e_{p c c q_{i}} \\
e_{p c c q_{i}} & -e_{p c c d_{i}}
\end{array}\right) \\
i_{C P L_{D Q i}}=\left(e_{C P L_{D Q i}}\right)^{-1} \cdot\left(\begin{array}{l}
P_{c i} \\
Q_{c i}
\end{array}\right)
\end{array}\right.
$$

\section{Simulations results}

To validate the efficiency of the strategies proposed in sections 2 and 3 for power sharing and synchronization, the multi-PCC mesh microgrid (Fig. 2) is modeled using the Simscape toolbox of Matlab/Simulation. DG1 and DG2 are formed with two controllable voltage sources Fig. 9, connected to PCC1 and PCC2 and controlled with the modified droop strategies described by equations (9) and (10). The main parameters of different element of the microgrid and its control are listed in table 1.

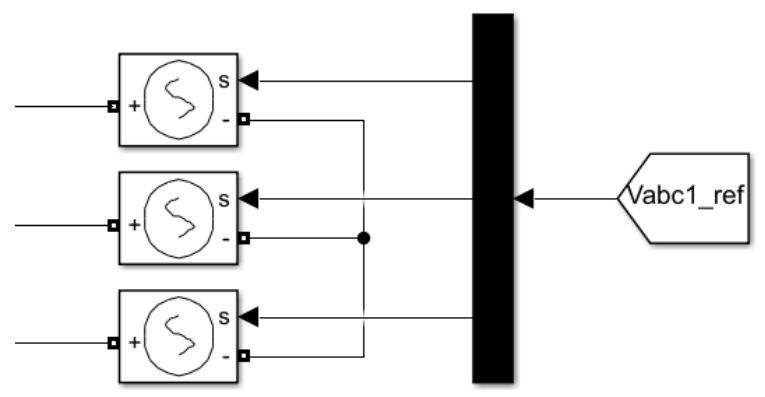

Fig. 9. Controllable voltage source.

Table 1. Parameters of the considered microgrid.

\begin{tabular}{|l|l|l|l|l|}
\hline Lines & $\begin{array}{c}\text { Resistance } \\
(\mathbf{\Omega})\end{array}$ & $\begin{array}{c}\text { Inductance } \\
(\mathbf{m H})\end{array}$ & $\begin{array}{c}\text { capacitance } \\
(\boldsymbol{\mu F})\end{array}$ & $\begin{array}{l}\text { Points of } \\
\text { connections }\end{array}$ \\
\hline Line 1 & 0.63 & 7.14 & 205 & Bus 8- Bus 7 \\
\hline Line 2 & 2.55 & 11.4 & 230 & Bus 5- Bus 7 \\
\hline Line 3 & 0.63 & 7.14 & 205 & Bus 8- Bus 9 \\
\hline Line 4 & 2 & 7 & 180 & Bus 9- Bus 6 \\
\hline Line 5 & 1.7 & 7.6 & 153.4 & Bus 4- Bus 5 \\
\hline Line 6 & 1.7 & 7.6 & 153.4 & Bus 4- Bus 6 \\
\hline $\begin{array}{c}\text { Sources } \\
\text { and } \\
\text { loads }\end{array}$ & $\begin{array}{c}\text { Active } \\
\text { power } \\
(\mathrm{MW})\end{array}$ & $\begin{array}{c}\text { Reactive } \\
\text { power } \\
(\text { Mvar })\end{array}$ & $\begin{array}{c}\text { Phase to } \\
\text { pahse voltage } \\
(\mathrm{kV})\end{array}$ & $\begin{array}{c}\text { Point of } \\
\text { connection }\end{array}$ \\
\hline $\begin{array}{l}\text { Source } \\
1\end{array}$ & 3 & 0.9 & 20 & Bus 7 \\
\hline $\begin{array}{l}\text { Source } \\
2\end{array}$ & 2 & 0.9 & 20 & Bus 9 \\
\hline Load 1 & 1.5 & 0.35 & 20 & Bus 5 \\
\hline Load 2 & 1.2 & 0.25 & 20 & Bus 6 \\
\hline Load 3 & 1 & 0.25 & 20 & Bus 8 \\
\hline
\end{tabular}

These simulations are performed to validate the efficiency of the synchronization and power sharing strategies proposed in relations (9) and (10). The first DG imposes at $0 \mathrm{~s}$ the frequency of the microgrid as well as the voltages of the different PCCs. The second DG is synchronized during the interval $[4 \mathrm{~s} \sim 5 \mathrm{~s}]$, and connected to the microgrid at $5 \mathrm{~s}$. Fig.10 and Fig.11 show the evolution of voltage amplitude and phase angle of both the $\mathrm{i}^{\text {th }}$ PCC $\left(\mathrm{V}_{p c c i}, \theta_{p c c i}\right)$ and the $\mathrm{i}^{\text {th }}$ DG $\left(\mathrm{V}_{D G i}, \theta_{D G i}\right)$ before and during the synchronization and after the interconnection of the $i^{\text {th }}$ DG. It can be seen that the synchronization is fast and it can be achieved in less than $1 \mathrm{s.}$

To prove that it is efficient and doesn't affect the power sharing Fig. 12 and Fig. 13 show the evolutions of active and reactive powers. It can be remarked in Fig.12 and Fig.13 that the active and reactive power sharing are ensured in steady state without being affected by the synchronization procedure. In addition, power peaks are cancelled during the transient state following the interconnection of the second DG to the microgrid. It should be noted that these performances are maintained with a higher number of DGs, even if the results are not presented.

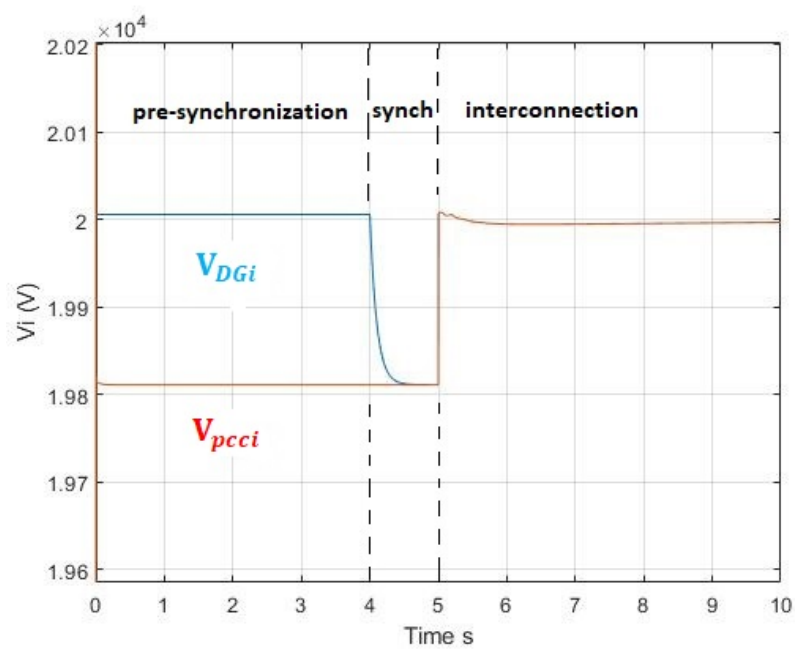

Fig. 10. Evolution of DG2 and PCC2 voltage amplitudes.

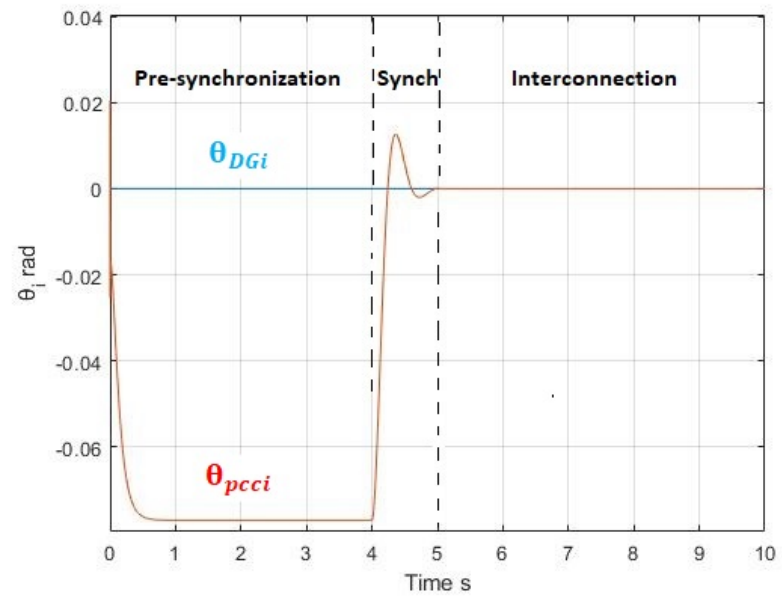

Fig. 11. Evolution of DG2 and PCC2 phase angles 


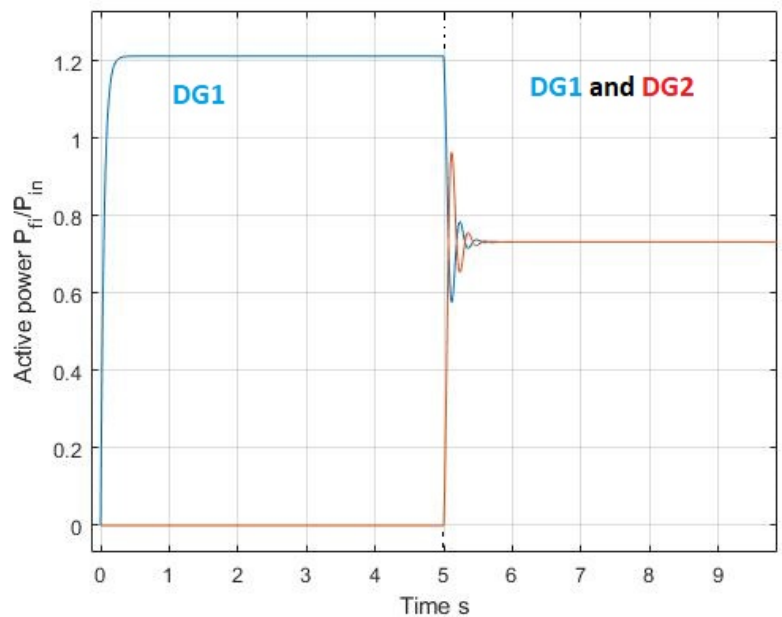

Fig. 12. Evolution of the DG active powers (first DG supplies the microgrid up to $5 \mathrm{~s}$, the second DG is connected to the microgrid at $5 \mathrm{~s}$ ).

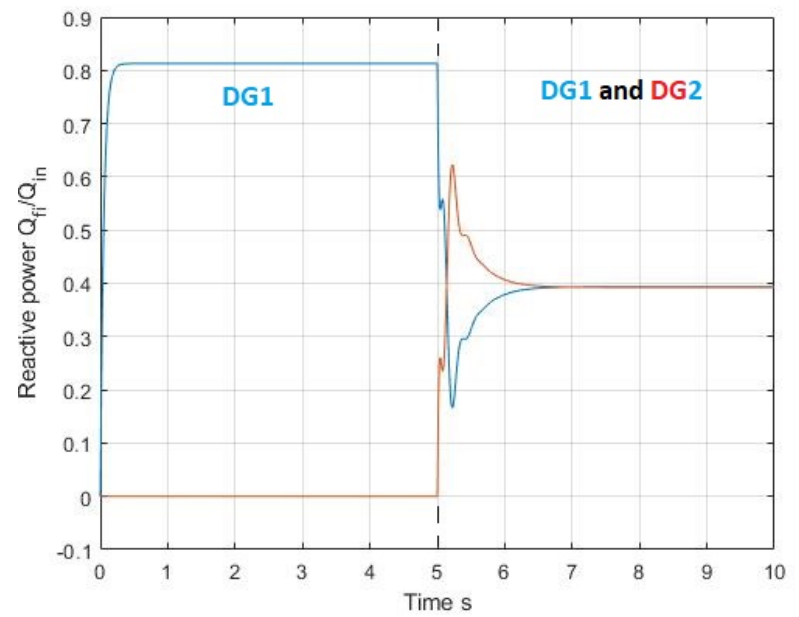

Fig. 13. Evolution of the DG reactive powers (first DG supplies the microgrid up to $5 \mathrm{~s}$, the second DG is connected to the microgrid at $5 \mathrm{~s}$ ).

To validate the developed model in section 4. A simulation is performed by applying the modified droop in (9) and (10) at the same time and under the same conditions on the Simscape model and the mathematical model in Matlab.

Figs. 14 and 15 show the evolution of the active and reactive powers of the two models on the same figure at the same time.

It can be seen that the evolutions of active and reactive powers in the two models are the same, which shows the validity of the model proposed in section 4 .

\section{Conclusion}

An improved Droop control strategy is proposed to ensure a controlled active and reactive power sharing in an islanded multi-PCC meshed microgrid. Only the RMS voltage measurement at a pilot node of the microgrid is necessary for its implementation. A synchronization technique is also introduced for the connection of the DGs to the microgrid. The proposed technique allows a correct connection without important power overflow during transient states. Moreover, the proposed droop control strategy with its synchronization technique is compliant with a "plug and play" function and does not require a priori knowledge of the microgrid structure and parameters. A mathematical model of the complete system has been proposed in order to perform stability and robustness studies. The developed modified droop control strategy can be adapted and used for power sharing in grid-connected mesh microgrids. The developed and validated state-space model can be the base for many future studies in mesh microgrids.

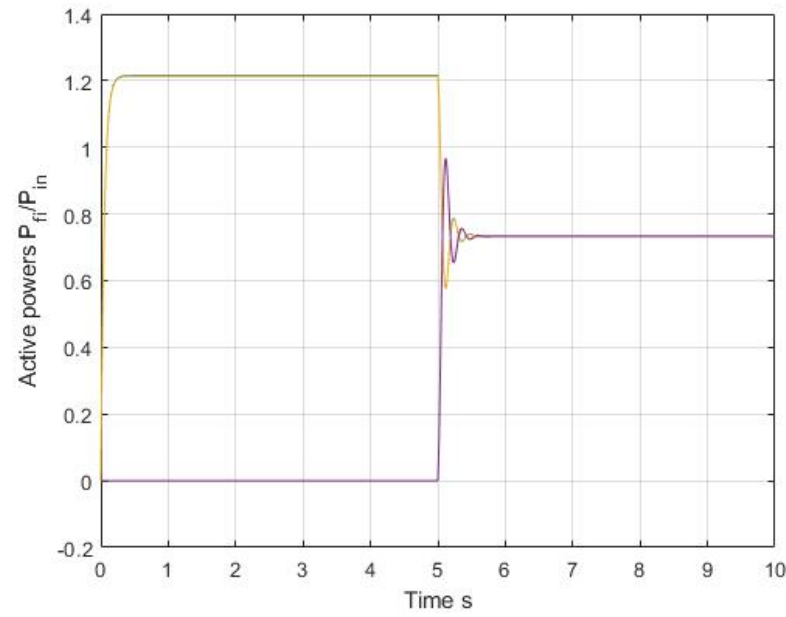

Fig. 14. Evolution of the DG active powers (Simscape model/mathematical model (first DG supplies the microgrid up to $5 \mathrm{~s}$, the second DG is connected to the microgrid at $5 \mathrm{~s}$ ).

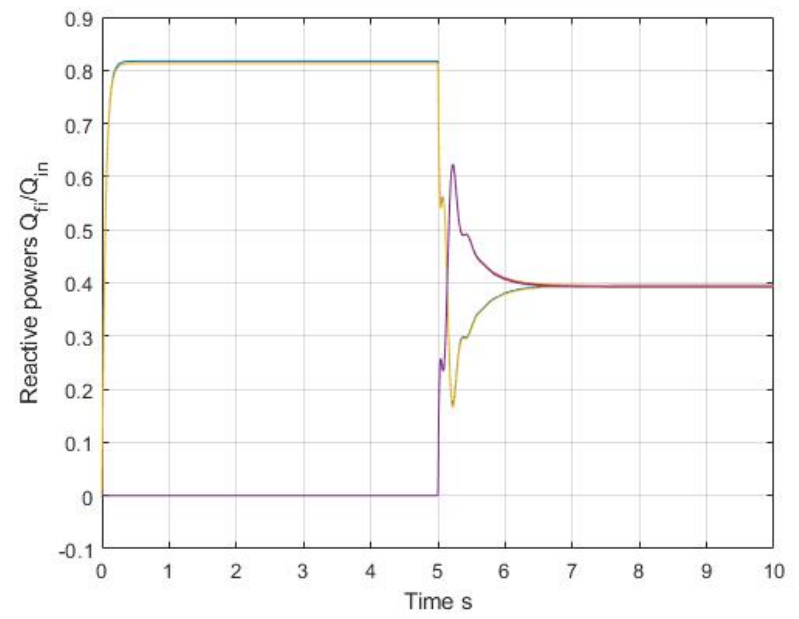

Fig. 15. Evolution of the DG reactive powers (Simscape model/mathematical model) (first DG supplies the microgrid up to $5 \mathrm{~s}$, the second DG is connected to the microgrid at $5 \mathrm{~s}$ ).

This project was financially supported by Ministry of Europe and Foreign Affairs, Ministry of Higher Education, Research and Innovation and the French Institute of Rabat (PHC TOUBKAL 2019 (French-Morocco bilateral program) Grant Number: $12345 \mathrm{AB}$ )

\section{References}

1. W. El-Khattam and M. Salama, "Distributed generation technologies, definitions and benefits," EPSR, vol. 71, no. 2, pp. 119-128, (2004). 
2. L. Zhang, L. Harnefors, and H. P. Nee, "Powersynchronization control of grid-connected voltagesource converters," IEEE Trans. on Power Systems, vol. 25, no. 12, pp. 809-820, (2010).

3. X. Haizhen, Z. Xing, L. Fang, Z. Debin, S. Rongliang, N. Hua, and C. Wei, "Synchronization strategy of microgrid from islanded to grid-connected mode seamless transfer," presented at IEEE International Conference of IEEE Region 10 (TENCON 2013), (2013).

4. U. B. Tayaba, M. A. B. Roslan, L. J. Hwai, and M. Kashif, "A review of droop control techniques for microgrid," RSER, vol. 76, pp. 717-727, Sept. (2017).

5. X. Xiaofei, L. Hong, and L. Zhipeng, "Research on new algorithm of droop control," presented at Chinese Control and Decision Conference (CCDC), (2013).

6. H. Han, X. Hou, J. Yang, J. Wu, M. Su, and J. M. Guerrero, "Review of power sharing control strategies for islanding operation of AC microgrids," IEEE Trans. on Smart Grid, vol. 7, no. 11, pp. 200215, (2016)

7. X. Huang, K. Wang, J. Qiu, L. Hang, G. Li, and X. Wang, "Decentralized control of multi-parallel gridforming DGs in islanded microgrids for enhanced transient performance," IEEE Access, vol. 7, pp. 17958-7968, Jan. (2019).

8. J. Peng, B. Fan, J. Duan, Q. Yang, and W. Liu, "Adaptive decentralized output-constrained control of single-bus DC microgrids," IEEE/CAA Journal of Automatica Sinica, vol. 6, no. 2, pp. 424-432, (2019).

9. H. Moussa, A. Shahin, J. P. Martin, S. Pierfederici, and N. Moubayed, "Optimal angle droop for power sharing enhancement with stability improvement in islanded microgrids," IEEE Trans. on Smart Grid, vol. 9, no. 15, pp. 5014-5026, (2017).

10. W. Yao, M. Chen, J. Matas, J. M. Guerrero, and Z. M. Qian, "Design and analysis of the droop control method for parallel inverters considering the impact of the complex impedance on the power sharing," IEEE Trans. on Industrial Electronics, vol. 58, no. 12, pp. 576-588, 2011.

11. Y. Zhu, F. Zhuo and H. Shi, "Accurate power sharing strategy for complex microgrid based on droop control method," 2013 IEEE ECCE Asia Downunder, Melbourne, VIC, 2013, pp. 344-350

12. Y. Hennane, J-P. Martin, A. Berdai, S. Pierfederici, and Farid Meibody-Tabar, "Power Sharing and Synchronization Strategies for Multiple PCC Islanded Microgrids," IJEETC, Vol. 9, No. 3, pp. 156-162, May (2020)

13. A. B. Jusoh, "The instability effect of constant power loads," in Proc. National Power and Energy Conf. PECon 2004, Nov. 2004, pp. 175-179.

14. M. Cespedes, L. Xing, and J. Sun, "Constant-power load system stabilization by passive damping," IEEE
Transactions on Power Electronics, vol. 26, no. 7, pp.1832-1836, Jul. 2011.

15. D. Marx, P. Magne, B. Nahid-Mobarakeh, S. Pierfederici, and B. Davat, "Large signal stability analysis tools in DC power systems with constant power loads and variable power loads - a review," IEEE Transactions on Power Electronics, vol. 27, no. 4, pp. 1773-1787, Apr. (2012). 